\title{
Cambios producidos por la adaptación al EEES en la carga de trabajo de estudiantes de Psicología
} Changes in Psychology students' workload due to alignment with the EHEA

\author{
Dra. Mª Inmaculada LÓPEZ-NÚÑEZ. Profesora Titular Interina. Universidad Complutense de Madrid \\ (mariai04@pdi.ucm.es).
}

Dra. Susana RUBIO-VALDEHITA. Profesora Titular. Universidad Complutense de Madrid (srubiova@ucm.es).

Dra. Eva Ma DÍAZ-RAMIRO. Profesora Contratada Doctora. Universidad Complutense de Madrid

(ediazram@ucm.es).

\section{Resumen:}

La adaptación al Espacio Europeo de Educación Superior (EEES) ha supuesto una importante modificación en la metodología docente y en el proceso de aprendizaje, lo que ha generado un cambio respecto a la carga de trabajo del alumnado. El principal objetivo de este estudio es evaluar y comparar la percepción de la carga de trabajo de estudiantes de Psicología de la licenciatura y del grado y analizar la naturaleza de los cambios producidos por esta adaptación. La muestra utilizada estuvo formada por 845 estudiantes (497 de licenciatura y 348 de grado). Para la medida de la carga percibida se empleó la escala NASA-TLX, que evalúa las siguientes dimensiones: demanda mental, demanda física, demanda temporal, rendimiento y frustración/insatisfacción; estas variables fueron evaluadas para las siguientes actividades académicas: asistencia a clases teóricas, asistencia a clases prácticas, rea- lización de trabajos en grupo fuera del aula, búsqueda de material y bibliografía, estudio y trabajo personal, asistencia a tutorías y otras actividades (seminarios, jornadas, etc.). Los resultados muestran un aumento significativo en la carga percibida de la asistencia a clases prácticas, en la realización de trabajos en grupo fuera del aula y en la carga total percibida para los estudiantes de grado. Se concluye que la adaptación al EEES ha supuesto un incremento en la percepción de carga de trabajo de los estudiantes, lo que apunta a la necesidad de trabajar en las aulas el desarrollo de competencias interpersonales y de planificación y organización del trabajo en equipo, así como una mayor coordinación docente entre los sistemas de evaluación.

Descriptores: Espacio Europeo de Educación Superior, carga de trabajo, NASA-TLX, estudiantes, psicología, Educación Superior.

Fecha de recepción de la versión definitiva de este artículo: 20-04-2018.

Cómo citar este artículo: López-Núñez, M. I., Rubio-Valdehita, S. y Díaz-Ramiro, E. (2019). Cambios producidos por la adaptación al EEES en la carga de trabajo de estudiantes de Psicología | Changes in Psychology students' workload due to alignment with the EHEA. Revista Española de Pedagogía, 77 (272), 163-176. doi: https://doi.org/10.22550/REP77-1-2019-02 


\section{Abstract:}

Alignment with the European Higher Education Area (EHEA) has involved changes in teaching methodology and in the learning process, which have led to a change in student workload. The main aim of this study is to evaluate and compare the perception of the workload of undergraduate and graduate psychology students and to analyse the nature of the changes produced by this adaptation. The sample comprised 845 students ( 497 readings for the licentiate's degree and 348 for the bachelor's degree). To measure perceived workload, the NASA-TLX scale was used. This evaluates the following dimensions: mental demand, physical demand, temporal demand, performance, and frustration/dissatisfaction; these variables were evaluated for the following academic activities: attending theoretical classes, attending practical classes, conducting group work outside the classroom, searching for material and bibliography, studying and personal work, attending tutorials, and other activities (seminars, conferences, etc.). The results show a significant increase in the perceived load of attendance in practical classes, in the performance of group work outside the classroom and in total perceived workload for bachelor's degree students. It is concluded that adjusting to the EHEA has involved an increase in perceived workload for students. This points to a need to work in the classroom on developing interpersonal skills and planning and organization of teamwork, as well as greater coordination between teachers in the evaluation systems.

Keywords: European Higher Education Area, workload, NASA-TLX, students, psychology, higher education.

\section{Introducción}

El Espacio Europeo de Educación Superior (EEES), derivado de la Declaración de Bolonia (1999), tiene entre sus objetivos la obtención de altas tasas de empleabilidad, así como mejorar la competitividad del Sistema de Educación Superior Europeo y promover la movilidad, en definitiva, promover una dimensión europea del sistema de educación superior (Ariza, Quevedo-Blasco, Bermúdez y Buela-Casal, 2013). Para alcanzar estos objetivos se propuso una transformación en la estructura de la enseñanza a través del Sistema Europeo de Transferencia y Acumulación de Crédi- tos (ECTS), un sistema que distribuye la carga de trabajo del estudiante con horas estimadas, por un lado, presenciales (clases, evaluaciones, etc.) y por otro, no presenciales con tutorización (trabajo dirigido y tutorías) y no presenciales autónomas (estudio y actividades individuales).

La adaptación al EEES ha conllevado importantes modificaciones tanto a nivel institucional como en los planes de estudios (Ariza, Bermúdez, Quevedo-Blasco y Buela-Casal, 2012; Ortega y Zych, 2013), así como en las metodologías docentes empleadas, incorporando métodos más ac- 
tivos (como asistencia a seminarios, resolución de problemas en equipo, realización de proyectos y presentaciones, etc.) que dotan al alumno de una mayor autonomía en un aprendizaje con menor carga presencial y mayor peso del trabajo personal. Estas modificaciones implican también un cambio en el proceso de enseñanza y aprendizaje más constructivista (Tejada y López, 2012), centrado en la adquisición de competencias y en el trabajo continuado del estudiante (De Miguel, 2006; Rodrigo y Almirón, 2013), lo que supone una mayor carga de trabajo no presencial (Díez, García e IPDDA, 2010; Ortiz et al., 2012).

Uno de los conceptos fundamentales implicados en los nuevos planes de estudio es la carga de trabajo del estudiante (Roca-Cuberes, 2013). Esta carga de trabajo se puede definir en función de las horas de trabajo que los estudiantes dedican a tareas académicas tanto dentro del centro educativo (asistencia a clases, tutorías, seminarios) como fuera del mismo (trabajos en grupo, estudio, consultas bibliográficas, etc.), es decir, se relacionaría con el tiempo necesario para completar un curso académico y adquirir las competencias establecidas como resultados del aprendizaje.

La bibliografía consultada coincide en señalar que ajustar la carga de trabajo a los créditos establecidos en los programas es una de las principales dificultades que tiene el profesorado (Reyes, Valdés y Castaño, 2006) y que el sistema ECTS supone un incremento en la carga de trabajo del alumnado (Castaño, Ruiz, Gómez-Alday y De Manuel, 2006; Roca-Cuberes, 2013; Rodríguez-Izquierdo, 2014).
A pesar de que esta reforma educativa se instauró oficialmente en 2010 , no disponemos de estudios empíricos en los que se analice el efecto de dicha adaptación desde la perspectiva de los estudiantes, menos aún en lo referente a la enseñanza de la Psicología (Ramiro-Sánchez, Bermúdez y Buela-Casal, 2016). En este sentido, hay una carencia evidente de estudios que exploren el impacto de dichos cambios en la percepción de carga de trabajo de los estudiantes del grado en Psicología en comparación con la de los estudiantes del sistema anterior (licenciatura). Por ejemplo, Ortiz et al. (2012) encuentran, desde una perspectiva basada en el estudio de los tiempos dedicados a cada tarea, diferencias en la carga de trabajo de estudiantes de licenciatura y grado y señalan que el cambio en las metodologías de enseñanza-aprendizaje debe abordarse desde la perspectiva del alumnado.

La carga de trabajo percibida por los estudiantes puede tener una influencia muy importante tanto sobre el nivel de rendimiento académico alcanzado como sobre el bienestar psicológico del alumnado, ya que es uno de los principales factores generadores de estrés académico (Cabanach, Souto-Gestal y Franco, 2016). Un exceso de carga de trabajo dificulta la adquisición de conocimientos y promueve un aprendizaje superficial (Lam, McNaught, Lee y Chan, 2012; Roca-Cuberes, 2013) fracaso y abandono académico (Cope y Staehr, 2005).

La carga de trabajo de los estudiantes ha sido evaluada desde diferentes perspectivas. Tradicionalmente, se ha relacionado con el cómputo de horas que los estudiantes dedi- 
can a las asignaturas incluidas en su titulación (Ruiz-Gallardo, Castaño, Gómez-Alday y Valdés, 2011), aunque en reducidos casos también se ha analizado la percepción de carga de trabajo de los estudiantes (Kyndt, Dochy, Struyven y Cascallar, 2011). Este segundo enfoque asume que la carga de trabajo percibida por el estudiante está modulada por diversos factores y, que es esta percepción, la que actúa como mayor factor generador de estrés (Ramsden, 1992). En esta línea, Kember y Leung (2006) encuentran que muchas horas de estudio no son percibidas como elevada sobrecarga cuando el diseño del curso es el adecuado y ponen de manifiesto la escasa relación entre el tiempo dedicado a una tarea y la percepción de carga (Hertzum y Holmegaard, 2013; Nosair y Handy, 2017).

Uno de los instrumentos más utilizados para evaluar la carga de trabajo percibida es la escala NASA-Task Load Index (TLX) (Hart y Staveland, 1988). González, Moreno y Garrosa (2005), Hart (2006) y Young, Zavelina y Hooper (2008) señalan las utilidades del NASA-TLX como un instrumento válido y fiable para el análisis de la carga de trabajo percibida en diferentes sectores: industrial, psicológico, sanitario, aeronáutico 0 trasportes. Sin embargo, en el ámbito educativo su utilización ha sido menos frecuente. LópezNúñez (2010) analiza la generalizabilidad y la validez de esta escala con una amplia muestra de estudiantes universitarios y concluye que se trata de un instrumento de utilidad para evaluar la carga de trabajo en el ámbito educativo. Kyndt et al. (2011) emplean el NASA-TLX para evaluar la carga de trabajo con el objetivo de analizar su papel mediador en la relación entre la motivación del estudiante y su estrategia de aprendizaje. Igualmente, Kurata, Bano y Matias (2015) utilizan el NASA-TLX en estudiantes de ingeniería, hallando una relación significativa entre la carga de trabajo y su rendimiento académico.

Casi dos décadas después de la Declaración de Bolonia (1999) e inmersos en las renovaciones de la acreditación de los títulos adaptados al EEES, parece adecuado llevar a cabo evaluaciones de los efectos de esta adaptación. El cambio parece que ha resultado positivo en cuanto a la inclusión de nuevas tecnologías (TIC) y metodologías que permiten una mayor interacción con el alumnado, sin embargo, otros aspectos como el incremento de la carga de trabajo, tanto para el profesorado como para el alumnado, no parecen tan positivos (Ariza et al., 2013; Quevedo-Blasco, Ariza y Buela-Casal, 2015).

Por todo ello, el objetivo de este estudio es analizar y comparar la percepción de carga de trabajo que tenían los estudiantes de psicología de la licenciatura (sistema anterior) con respecto a la de los estudiantes del nuevo sistema adaptado al EEES (grado), con el fin de investigar la naturaleza de los cambios producidos en esta variable.

\section{Método \\ 2.1. Participantes}

La muestra estuvo formada por 845 estudiantes de tercer curso de Psicología de la Universidad Complutense de 
Madrid, de los cuales 497 eran de licenciatura y 348 de grado. En cuanto a la distribución por sexo, cursando la licenciatura había 415 mujeres $(83.5 \%)$ y 82 varones (16.5\%), y en grado 282 eran mujeres $(81 \%)$ y 66 varones $(19 \%)$. La media de edad de los estudiantes de licenciatura fue de 21.63 años $(D T=3.33)$ y de los de grado de 21.85 ( $D T=3.53)$. Los datos se recogieron en una única sesión durante una de las clases del tercer curso, tanto de grado como de licenciatura, siguiendo un muestreo de tipo incidental.

\subsection{Instrumento}

Para evaluar la carga de trabajo se empleó la escala NASA-TLX (Hart y Staveland, 1998). Este instrumento distingue seis dimensiones de carga de trabajo, cada una de las cuales es evaluada por los individuos en una escala de 0 a 100. Las dimensiones son las siguientes: esfuer$z o$ (grado de esfuerzo general, mental y físico que debe realizar para obtener un adecuado nivel de rendimiento), demanda mental (cantidad de actividad mental y perceptiva que requiere la tarea), demanda física (cantidad de actividad física que requiere la tarea), demanda temporal (nivel de presión temporal percibida), rendimiento (grado de satisfacción con el propio nivel de rendimiento alcanzado en la tarea) y frustración/insatisfacción (grado de inseguridad, irritación o descontento sentido durante la realización de la tarea). El estudiante debía evaluar el grado en que cada una de sus actividades académicas le requiere cada una de las dimensiones de carga de trabajo. Se distinguieron siete actividades académi- cas: asistir a clases teóricas, asistir a clases prácticas, realización de trabajos en grupo fuera del aula, búsqueda de material y bibliografía, estudio y trabajo personal, asistencia a tutorías y otras actividades (asistencia a seminarios, cursos, jornadas, etc.).

La elección de estas actividades se realizó pensando en los cambios e importancia dados por el EEES al trabajo autónomo del alumnado y la adquisición de competencias, tanto las específicas de la titulación como las transversales, para su inserción en el contexto socio-laboral (ANECA, 2005).

Con las valoraciones proporcionadas por cada estudiante se calculó un índice de carga de trabajo global para cada actividad mediante el cómputo de la media aritmética de las puntuaciones en cada dimensión (Hart, 2006).

Además, todos los participantes contestaron un breve cuestionario demográfico mediante el que se recogían datos relativos a su sexo y edad.

\subsection{Diseño y Procedimiento}

Se trata de un estudio de tipo no experimental y ex post facto. La escala NASA-TLX, inicialmente, en su fase de aplicación, constaba de dos fases: la de ponderación y la de puntuación 0 valoración. La fase de ponderación pretendía conocer la concepción inicial de carga de trabajo que tenían los individuos y la importancia relativa de cada una de las seis dimensiones en dicha conceptualización. Sin embargo, numerosos estudios han 
puesto de manifiesto que no es necesario llevar a cabo la fase de ponderación (López, Rubio, Martín y Luceño, 2010), encontrando valores de correlación elevados entre las puntuaciones ponderadas y las no ponderadas (entre .93 y .97). Por este motivo, solo se llevó a cabo la fase de valoración. Todos los estudiantes participaron de forma anónima y completamente voluntaria, habiendo firmado previamente un consentimiento aceptando su participación en el estudio y la utilización de los datos con fines exclusivamente de investigación.

\subsection{Análisis estadístico}

Todos los análisis fueron realizados con el paquete estadístico Statistical Package for the Social Science (SPSS), versión 22.0. En primer lugar, se calcularon los estadísticos descriptivos de todas las variables consideradas. A continuación, se comprobó la equivalencia de los dos grupos en cuanto a su media de edad y la distribución por sexo mediante el cálculo del estadístico $\chi^{2}$ (para el sexo) y del estadístico $t$ de student (para la edad). Posteriormente se realizaron análisis de comparación de medias de las puntuaciones de carga de trabajo obtenidas por los dos grupos (licenciatura vs. grado) en cada una de las actividades académicas. Para ello, se calculó el valor del estadístico t de student, su nivel de significación $p$ (se fija en .05) y $\eta^{2}$ parcial para comprobar el tamaño del efecto.

\section{Resultados}

En primer lugar, se calcularon las medias y desviaciones típicas (DT) de las pun- tuaciones de carga (para cada dimensión de carga y para la puntuación global) en ambos grupos (licenciatura y grado) (Tabla 1). La puntuación de carga total fue obtenida promediando todas las valoraciones de carga de todas las dimensiones y actividades.

Los resultados mostraron la equivalencia de los grupos en cuanto a la distribución por sexo $\left(\chi^{2}=1.70, p=.430\right)$ y en cuanto a la media de edad $[t(913)=-0.66$, $p=.508$ ], ya que ambos estadísticos resultaron no significativos.

Una vez comprobado el cumplimiento del supuesto de homogeneidad de varianzas mediante la prueba de Levene ( $p>.05$ en todos los casos), se realizaron las comparaciones de medias de las puntuaciones de carga global de cada actividad según la titulación. Los resultados de este análisis aparecen en la Tabla 1. El análisis de esta tabla permite concluir que los estudiantes de grado perciben una mayor carga que los de licenciatura en todas las actividades consideradas excepto en «estudio y trabajo personal». Alcanzaron la significación estadística las diferencias en las actividades «realización de trabajos en grupo fuera del aula» $\mathrm{y}$ «asistencia a clases prácticas», así como en las puntuaciones de carga total. En las tres actividades, el grupo de estudiantes de grado mostró puntuaciones de carga significativamente más elevadas. Los valores de $\eta^{2}$ muestran que el tamaño del efecto es más elevado para la actividad «realización de trabajos en grupo fuera del aula», lo que indica que es en esta actividad en la que la diferencia es más evidente. 
TABLA 1. Media (M) y desviación típica (DT) de la carga de trabajo global de cada una de las actividades y resultados de la comparación de medias entre titulaciones.

\begin{tabular}{|l|c|c|c|c|c|}
\cline { 2 - 6 } \multicolumn{1}{c|}{} & Licenciatura & Grado & & \\
\cline { 2 - 6 } \multicolumn{1}{l|}{} & $\mathrm{M}(\mathrm{DT})$ & $\mathrm{M}(\mathrm{DT})$ & $t(843)$ & $p$ & $\eta^{2}$ \\
\hline Asistencia a clases teóricas & $\begin{array}{c}52.86 \\
(12.29)\end{array}$ & $\begin{array}{c}53.35 \\
(13.09)\end{array}$ & -0.55 & .581 & .000 \\
\hline Asistencia a clases prácticas & $\begin{array}{c}47.80 \\
(13.82)\end{array}$ & $\begin{array}{c}49.80 \\
(13.00)\end{array}$ & -2.11 & .035 & .005 \\
\hline Realización de trabajos en grupo & $\begin{array}{c}49.63 \\
(14.81)\end{array}$ & $\begin{array}{c}55.01 \\
(13.44)\end{array}$ & -5.38 & .000 & .033 \\
\hline fuera del aula & $\begin{array}{c}40.28 \\
(15.23)\end{array}$ & $\begin{array}{c}40.62 \\
(14.51)\end{array}$ & -0.32 & .744 & .000 \\
\hline y bibqueda de material & $\begin{array}{c}57.05 \\
(12.51)\end{array}$ & $\begin{array}{c}56.79 \\
(11.50)\end{array}$ & 0.31 & .756 & .000 \\
\hline Estudio y trabajo personal & $\begin{array}{c}25.68 \\
(11.82)\end{array}$ & $\begin{array}{c}26.97 \\
(12.87)\end{array}$ & -1.49 & .135 & .003 \\
\hline Asistencia a tutorías & $\begin{array}{c}28.72 \\
(14.46)\end{array}$ & $\begin{array}{c}29.42 \\
(14.29)\end{array}$ & -0.68 & .497 & .001 \\
\hline $\begin{array}{l}\text { Otras actividades } \\
\text { (seminarios, congresos, etc.) }\end{array}$ & $\begin{array}{c}43.37 \\
(9.20)\end{array}$ & $\begin{array}{c}44.58 \\
(8.96)\end{array}$ & -1.89 & .048 & .005 \\
\hline \multicolumn{1}{|c|}{ Carga Total } & & & & \\
\hline
\end{tabular}

Fuente: Elaboración propia.

Con el objetivo de analizar en más detalle qué dimensión o dimensiones de carga de trabajo eran la causa de estas diferencias, se realizaron comparaciones de medias entre grado y licenciatura en cada una de las 6 dimensiones del NASA-TLX para las actividades en las que se encontraron resultados significativos en el análisis anterior.

Los resultados de este análisis aparecen en la Tabla 2.

Para la actividad «asistencia a clases prácticas», los resultados muestran diferencias elevadas y estadísticamente significativas solamente en la dimensión demanda mental, lo cual indica que los estudiantes de grado perciben esta actividad académica como bastante más demandan- te desde un punto de vista cognitivo que los estudiantes de licenciatura.

Para la actividad «trabajos en grupo fuera del aula» los resultados muestran mayores diferencias que en la actividad anterior. En este caso, las diferencias entre ambos grupos resultaron estadísticamente significativas en todas las dimensiones excepto en la de rendimiento. Estos resultados indicarían que la realización de trabajos en grupo fuera del horario de clases es una actividad mucho más demandante a todos los niveles para los estudiantes de grado, quienes tendrían que esforzarse más para conseguir niveles muy similares de rendimiento. Esto les genera a su vez sentimientos de frustración más acusados. Resultados en la misma línea son los que se muestran para la puntuación de carga total. 
TABLA 2. Media (M), desviación típica (DT) y resultados de la comparación de medias entre ambas titulaciones en las puntuaciones de carga de cada dimensión del NASA-TLX.

Asistencia a clases prácticas fuera del aula

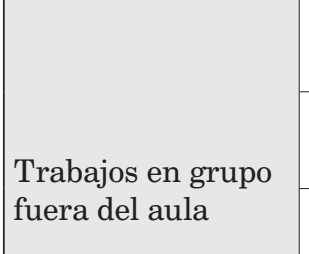

\begin{tabular}{|c|c|c|c|c|c|}
\hline & Licenciatura & Grado & & & \\
\hline & $\mathrm{M}(\mathrm{DT})$ & $\mathrm{M}(\mathrm{DT})$ & $t(843)$ & $p$ & $\eta^{2}$ \\
\hline Esfuerzo & $\begin{array}{c}67.08 \\
(26.26)\end{array}$ & $\begin{array}{c}67.43 \\
(25.24)\end{array}$ & -0.30 & .764 & .000 \\
\hline D. Mental & $\begin{array}{c}59.89 \\
(22.60)\end{array}$ & $\begin{array}{c}63.67 \\
(20.43)\end{array}$ & -2.55 & .011 & .007 \\
\hline D. Física & $\begin{array}{c}43.05 \\
(26.41)\end{array}$ & $\begin{array}{c}45.55 \\
(27.48)\end{array}$ & -1.38 & .165 & .002 \\
\hline D. Temporal & $\begin{array}{c}57.70 \\
(27.10)\end{array}$ & $\begin{array}{c}60.34 \\
(26.62)\end{array}$ & -1.40 & .161 & .002 \\
\hline Rendimiento & $\begin{array}{c}29.71 \\
(21.01)\end{array}$ & $\begin{array}{c}28.82 \\
(18.73)\end{array}$ & 0.52 & .597 & .000 \\
\hline Frustración & $\begin{array}{c}29.87 \\
(25.53)\end{array}$ & $\begin{array}{c}32.90 \\
(25.95)\end{array}$ & -1.67 & .094 & .003 \\
\hline Esfuerzo & $\begin{array}{c}63.80 \\
(25.16)\end{array}$ & $\begin{array}{c}71.61 \\
(21.48)\end{array}$ & -4.68 & .000 & .026 \\
\hline D. Mental & $\begin{array}{c}63.01 \\
(23.43)\end{array}$ & $\begin{array}{c}71.59 \\
(20.86)\end{array}$ & -5.52 & .000 & .034 \\
\hline D. Física & $\begin{array}{c}44.38 \\
(26.36)\end{array}$ & $\begin{array}{c}49.42 \\
(28.96)\end{array}$ & -2.59 & .010 & .008 \\
\hline D. Temporal & $\begin{array}{c}60.92 \\
(25.59)\end{array}$ & $\begin{array}{c}66.71 \\
(23.58)\end{array}$ & -3.20 & .001 & .013 \\
\hline Rendimiento & $\begin{array}{c}31.46 \\
(20.71)\end{array}$ & $\begin{array}{c}29.64 \\
(19.92)\end{array}$ & 1.26 & .206 & .002 \\
\hline Frustración & $\begin{array}{c}34.56 \\
(27.72)\end{array}$ & $\begin{array}{c}41.63 \\
(26.85)\end{array}$ & -3.68 & .000 & .016 \\
\hline Esfuerzo & $\begin{array}{c}52.36 \\
(14.69) \\
\end{array}$ & $\begin{array}{c}56.27 \\
(16.14) \\
\end{array}$ & -3.57 & .000 & .016 \\
\hline D. Mental & $\begin{array}{c}51.27 \\
(14.71)\end{array}$ & $\begin{array}{c}58.61 \\
(16.28)\end{array}$ & -6.48 & .000 & .047 \\
\hline D. Física & $\begin{array}{c}36.04 \\
(19.02) \\
\end{array}$ & $\begin{array}{c}39.19 \\
(19.96) \\
\end{array}$ & -2.40 & .016 & .006 \\
\hline D. Temporal & $\begin{array}{c}50.23 \\
(15.92)\end{array}$ & $\begin{array}{c}53.49 \\
(15.78) \\
\end{array}$ & -2.89 & .004 & .010 \\
\hline Rendimiento & $\begin{array}{c}42.58 \\
(14.80)\end{array}$ & $\begin{array}{c}40.97 \\
(14.30)\end{array}$ & 1.57 & .116 & .003 \\
\hline Frustración & $\begin{array}{c}27.76 \\
(16.86)\end{array}$ & $\begin{array}{c}31.44 \\
(17.35)\end{array}$ & -3.13 & .002 & .011 \\
\hline
\end{tabular}

Fuente: Elaboración propia. 


\section{Discusión}

Dada la escasez de trabajos de investigación en los que se haya analizado la percepción que tienen los estudiantes de las exigencias académicas en la adaptación de la titulación al EEES, el propósito del presente estudio fue evaluar los niveles de carga de trabajo en dos muestras de estudiantes de Psicología (de grado y de licenciatura) y analizar si los cambios introducidos por la adaptación al EEES han producido alguna variación significativa en la percepción de carga de trabajo de los estudiantes.

La utilización de la escala NASA-TLX ha permitido no solo conocer los niveles de carga percibidos por los estudiantes, sino además ahondar en el conocimiento de qué actividades son las que generan mayores niveles de carga de trabajo.

Los resultados del presente estudio indican que existen diferencias en la percepción de carga total de trabajo entre ambos grupos, obteniendo puntuaciones más elevadas el grupo de grado (adaptado al EEES) que el de licenciatura (sistema anterior). Analizando los datos por tareas específicas, se encontraron diferencias estadísticamente significativas en las actividades «asistencia a clases prácticas» y «realización de trabajos en grupo fuera del aula».

Analizando más detalladamente cada una de ellas, observamos que en la tarea «asistencia a las clases prácticas» se encuentran diferencias estadísticamente significativas en la dimensión demanda mental, por lo que esta actividad gene- ra un mayor esfuerzo cognitivo al grupo de grado que al de licenciatura. La actividad «realización de trabajos en grupo fuera del aula» es percibida como mucho más demandante que la anterior, encontrándose diferencias significativas en todas las dimensiones excepto en rendimiento, lo que significa que los alumnos de grado tienen que hacer mayores esfuerzos para conseguir unos resultados similares, lo que además de mayores demandas provoca sentimientos de frustración.

Estos resultados permiten extraer dos conclusiones importantes de la adaptación al EEES sobre las que es necesario reflexionar y que abren perspectivas de investigación futura. La primera, es la evidencia de una mayor carga de trabajo percibida por el alumnado en la nueva titulación de Psicología tras la implementación del grado y la segunda, es que la actividad que mayor carga genera en todas sus dimensiones es la de «realización de trabajos en grupo fuera del aula».

Con respecto a la primera consideración, los resultados encontrados coinciden con los obtenidos por otros autores en cuanto a un incremento en la percepción de carga de trabajo en los estudiante de grado con respecto a los de licenciatura (Roca-Cuberes, 2013; Rodríguez-Izquierdo, 2014). Para explicar este resultado, diversas investigaciones señalan que la carga de trabajo en las titulaciones de grado no es mayor que la que corresponde según el número de créditos y que la percepción de incremento de carga, por parte del alumnado, se podría deber a que las nuevas me- 
todologías obligan a una distribución temporal continua a lo largo del cuatrimestre (Julián et al., 2010). Otros autores señalan que la falta de relación entre dedicación y rendimiento tampoco se podría explicar por una mayor carga de trabajo, sino por la falta de técnicas apropiadas de estudio, el escaso uso que se hace de recursos de aprendizaje como la tutoría personalizada 0 la necesidad de un mayor esfuerzo del alumnado (Ortiz et al., 2012). Este incremento de la percepción de carga de trabajo puede ser debido a que el EEES establece un sistema de enseñanza y aprendizaje autónomo, que exige unas estrategias de organización y planificación con las que los estudiantes no estaban familiarizados ni preparados.

Investigaciones futuras deberían evaluar la percepción actual de los niveles de carga y hacer estudios longitudinales para conocer si los nuevos grados están consiguiendo la adquisición de un aprendizaje más proactivo y una disminución de la percepción de carga a lo largo de los cursos académicos. Sus resultados servirán para conocer cómo ha sido el proceso de adaptación al EEES y también para plantear intervenciones en estrategias y hábitos de estudio, aplicando los conocimientos procedentes de la pedagogía con el fin de facilitar y mejorar el proceso de aprendizaje.

En cuanto a la segunda consideración, esto es, que la actividad «trabajo en grupo fuera del aula» es la que mayor carga percibida produce, estaría indicando la dificultad que el alumnado tiene para adaptarse a un sistema de aprendizaje que implica el desarrollo de competencias interpersonales y de planificación y organización. Además, es señalada como una actividad estresante, que les demanda tiempo y cuyo mayor esfuerzo no se corresponde con la recompensa esperada.

El EEES señala como una de las principales competencias trasversales la de trabajo en equipo. Varios estudios (Alonso-Martín, 2010; Arce, Fariña, Novo y Seijo, 2012; París, Torrelles y Mas, 2016) han evaluado la adquisición y desarrollo de esta competencia en estudiantes universitarios. Los resultados de estas investigaciones muestran que los estudiantes no consideran esta competencia como un recurso que les ayude en su aprendizaje, sino que la perciben como un factor estresante y que, en general, no está siendo adquirida por el alumnado. Como señalan Fidalgo-Blanco, Leris, Sein-Echaluce y García-Peñalvo (2013), la aplicación del trabajo en equipo en el entorno académico se suele realizar en lo que los autores denominan modelo "caja negra», es decir, lo habitual es que no se evalúe la propia competencia de trabajo en equipo sino solamente la calidad del trabajo una vez finalizado. Se deja, por tanto, a los estudiantes que desarrollen la competencia de trabajo en equipo por mera exposición, sin dotarles de recursos ni de orientación alguna, asumiendo erróneamente que los estudiantes acceden a los grados universitarios con esta competencia ya adquirida previamente en la enseñanza no universitaria.

Los resultados encontrados en este estudio ponen de manifiesto la necesidad de desarrollar programas de formación tanto 
del alumnado como de los docentes para el desarrollo y evaluación de esta competencia transversal, ya que, precisamente, es una de las más importantes entre las señaladas en el perfil profesional de graduado en Psicología (ANECA, 2005).

En cuanto a las limitaciones del estudio hay que señalar que se trata de una muestra de tipo incidental referida a una sola universidad, por lo que un mayor tamaño muestral procedente de distintas universidades españolas hubiera permitido una mayor generalización de los resultados obtenidos.

Por último, entre las principales implicaciones prácticas de los resultados obtenidos, cabe señalar que se trata de un estudio pionero en la evaluación de los niveles de carga de trabajo en la adaptación del título de grado en Psicología al EEES. Se confirma, en línea con la literatura consultada, que esta adaptación supuso un incremento en los niveles de carga de trabajo percibidos en los estudiantes y que la actividad de trabajo en equipo es percibida como muy demandante y frustrante. Estos resultados permiten abrir nuevas líneas de investigación en cuanto a las diferencias detectadas y plantear futuros estudios, considerando la percepción de carga de trabajo como un indicador de calidad y de evaluación del proceso de adaptación al EEES.

\section{Referencias bibliográficas}

Agencia Nacional de Evaluación de la Calidad y Acreditación, (ANECA). (2005). Libro blanco del título de grado en Psicología. Recuperado de https://goo.gl/Lucfeq (Consultado el 29/3/2017).
Alonso-Martín, P. (2010). La valoración de la importancia de las competencias transversales: comparación de su percepción al inicio y final de curso en alumnos de psicología. Revista de investigación educativa, 28 (1), 119-140.

Arce, R., Fariña, F., Novo, M. y Seijo, D. (2012). Efecto del sistema de enseñanza en el rendimiento académico, burnout experimentado y estrés académico. Aula abierta, 40 (2), 3-10.

Ariza, T., Bermúdez, M. P., Quevedo-Blasco, R. y Buela-Casal, G. (2012). Evolución de la legislación de doctorado en los países del EEES. Revista Iberoamericana de Psicología y Salud, 3 (2), 89-108.

Ariza, T., Quevedo-Blasco, R., Bermúdez, M. P. y Buela-Casal, G. (2013). Analysis of postgraduate programs in the EHEA and the USA. Revista de Psicodidáctica, 18 (1), 197-219. doi: https:// doi.org/10.1387/RevPsicodidact.5511

Cabanach, R. G., Souto-Gestal, A. y Franco, V. (2016). Escala de Estresores Académicos para la evaluación de los estresores académicos en estudiantes universitarios. Revista Iberoamericana de Psicología y Salud, 7 (2), 41-50.

Castaño, S., Ruiz, J. R., Gómez-Alday, J. J. y De Manuel, T. (2006). Adaptación metodológica al EEES: resultados de una experiencia. Revista de la Red-U, 5, 35-48.

Cope, C. y Staehr, L. (2005). Improving students' learning approaches through intervention in an information systems learning environment. Studiesin HigherEducation, 30 (2),181-197.doi: https://doi.org/10.1080/03075070500043275

De Miguel, M. (Ed.). (2006). Metodología de enseñanza y aprendizaje para el desarrollo de competencias. Madrid: Alianza editorial.

Declaración de Bolonia (1999). El espacio Europeo de la enseñanza superior. Declaración conjunta de los ministros europeos de educación. Recuperado de https://goo.gl/aoXldD (Consultado el 20/3/2017)

Díez, M. C., García, J. N. e IPDDA (2010). Percepción de metodologías docentes y desarrollo de competencias al EEES. Boletín de Psicología, 99, 45-69.

Fidalgo-Blanco, Á., Lerís, D., Sein-Echaluce, M. L. y García-Peñalvo, F. J. (2013). Indicadores para el seguimiento y evaluación de la compe- 
tencia de trabajo en equipo a través del método CTMTC. Trabajo presentado en el II Congreso Internacional sobre Aprendizaje, Innovación y Competitividad (CINAIC 2013). Recuperado de https://goo.gl/1aiZBq (Consultado el 19/9/2017).

González, J. L., Moreno, B. y Garrosa, E. (2005). Carga mental y fatiga laboral. Madrid: Pirámide.

Hart, S. G. (2006). NASA-Task Load Index (NASA-TLX); 20 years later. Proceedings of the Human Factors and Ergonomics Society Annual Meeting, 50 (9), 904-908. doi: https://doi. org/10.1177/154193120605000909

Hart, S. G. y Staveland, L. E. (1988). Development of NASA-TLX (Task LoadIndex): Results of empirical and theoretical research. En P. A. Hancock y N. Meshkati (Eds.), Human mental workload (pp. 139-183). Amsterdam: North-Holland.

Hertzum, M. y Holmegaard, K. D. (2013). Perceived time as a measure of mental workload: Effects of time constraints and task success. International Journal of Human-Computer Interaction, 29 (1), 26-39. doi: https://doi.org/10.1 080/10447318.2012.676538

Julián, J., Zaragoza, J., Castejón, F. J. y López, V. M. (2010). Carga de trabajo en diferentes asignaturas que experimentan el sistema ECTS. Revista Internacional de Medicina y Ciencias de la Actividad Fisica y del Deporte, 10 (38), 218-233.

Kember, D. y Leung, D. Y. (2006). Characterising a teaching and learning environment conducive to making demands on students while not making their workload excessive. Studies in Higher Education, 31 (2), 185-198. doi: https:// doi.org/10.1080/03075070600572074

Kyndt E., Dochy F., Struyven K. y Cascallar E. (2011). The direct and indirect effect of motivation for learning on students' approaches to learning through the perceptions of workload and task complexity. Higher Education Research \& Development, 30 (2), 135-150. doi: https://doi.org/10.1080/07294360.2010.501329

Kurata, Y. B., Bano, R. M. L. P. y Matias, A. C. (2015). Effects of workload on academic performance among working students in an un- dergraduate engineering program. Procedia Manufacturing, 3, 3360-3367. doi: https://doi. org/10.1016/j.promfg.2015.07.497

Lam, P., McNaught, C., Lee, J. y Chan, M. (2012). The impact of student workload on learning experiences. Hong Kong: Centre for Learning Enhancement and Research, the Chinese University of Hong Kong. Recuperado de https:// goo.gl/LCg2G8 (Consultado el 02/10/2017).

López, I., Rubio, S., Martín, J. y Luceño, L. (2010). Fase de ponderación del NASA-TLX: iun paso innecesario en la aplicación del instrumento? Edupsykhé: Revista de Psicología y Educación, 9 (2), 159-176.

López-Núñez, M. I. (2010). Generalización al ámbito laboral de dos instrumentos de medida subjetiva de la carga mental (Tesis doctoral). Universidad Complutense de Madrid. Recuperado de https://goo.gl/MPIBKT (Consultado el 26/4/2017).

Nosair, E. y Hamdy, H. (2017). Total Student Workload: Implications of the European Credit Transfer and Accumulation System for an Integrated, Problem-Based Medical Curriculum. Health Professions Education, 3 (2), 99-107. doi: https://doi.org/10.1016/j.hpe.2017.01.002

Ortega, R. y Zych, I. (2013). Profesionales de la Psicología y formación universitaria: buscando referencias para el título de grado en Psicología. International Journal of Psychology and Psychological Therapy, 13 (1), 83-96.

Ortíz, V. M., Jenaro, C., Meilán, J. G., Zubiauz, B., Mayor, M. A. y Arana, J. M. (2012). Carga de Trabajo en el EEES: La necesidad de coordinación docente entre asignaturas. En J. D. Álvarez, M. T. Tortosa y N. Pellín (Coords.), IX Jornadas de Redes de Investigación en Docencia Universitaria. Diseño de buenas prácticas docentes en el contexto actual, 1863-1877. Recuperado de https://goo.gl/THFgLK

París, G., Torrelles, C. y Mas, O. (2016). La evaluación de la competencia "trabajo en equipo» de los estudiantes universitarios. Revista d'Innovació Docent Universitària, 8, 86-97. doi: https://doi.org/10.1344/RIDU2016.8.10

Quevedo-Blasco, R., Ariza, T. y Buela-Casal, G. (2015). Evaluación de la satisfacción del profesorado de ciencias con la adaptación al Espa- 
cio Europeo de Educación Superior. Educación XXI, 18 (1), 45-70. doi: https://doi.org/10.5944/ educXX1.18.1.12311

Ramiro-Sánchez, T., Bermúdez, M. P. y Buela-Casal, G. (2016). Titulación de Psicología en el EEES: comparación entre licenciados y graduados españoles. Revista de Psicodidáctica, 21 (1), 175-189. doi: https://doi.org/10.1387/ RevPsicodidact.13923

Ramsden P. (1992). Learning to teach in higher education. Londres, UK: Routledge.

Reyes, J. R., Valdés, A. y Castaño, S. (2006). Prácticum y carga de trabajo. Revista deInvestigación Educativa, 24 (2), 557-574.

Rodríguez-Izquierdo, R. M. (2014). Modelo formativo en el Espacio Europeo de Educación Superior: valoraciones de los estudiantes. Aula Abierta, 42 (2), 106-113. doi: https://doi. org/10.1016/j.aula.2014.03.002

Roca-Cuberes, C. (2013). La percepción de los estudiantes sobre su experiencia de aprendizaje a partir de su dedicación temporal y adquisición de competencias: un estudio sobre la implementación del Grado de Traducción e Interpretación de la Universidad Pompeu Fabra. Revista Complutense de Educación, 24 (2), 359-379.

Rodrigo, M. y Almiron, N. (2013). Autopercepción de la adquisición de competencias de los estudiantes de periodismo: el caso de la Universitat Pompeu Fabra. Aula Abierta, 41 (1), 99-110.

Ruiz-Gallardo J. R., Castaño, S., Gómez-Alday J. J. y Valdés, A. (2011). Assessing student workload in problem based learning: relationships among teaching method, student workload and achievement. A case study in Natural Sciences. Teaching and Teacher Education, 27 (3), 619-627. doi: http://doi.org/10.1016/j.tate.2010.11.001

Tejada, Á. y López, M. (2012). Nuevas metodologías docentes en los títulos de grado: la literatura como recurso pedagógico colaborativo. Aula Abierta, 40 (3), 107-114.

Young, G., Zavelina, L. y Hooper, V. (2008). Assessment of workload using NASA task load index in perianesthesia nursing. Journal of Perianesthesia Nursing, 23 (2), 102-110. doi: https://doi. org/10.1016/j.jopan.2008.01.008

\section{Biografía de las autoras}

María Inmaculada López-Núñez es Doctora en Psicología por la Universidad Complutense de Madrid y Profesora del Departamento de Psicología Social, del Trabajo y Diferencial de esta misma universidad. Imparte docencia en el grado de Psicología y en el máster en Formación del Profesorado, en la especialidad de Formación y Orientación Laboral, en la Facultad de Educación. Sus principales líneas de investigación son: psicología ocupacional, desarrollo y adquisición de competencias profesionales, innovación y emprendimiento.

iD https://orcid.org/0000-0001-9179-7219

Susana Rubio-Valdehita es Doctora en Psicología por la Universidad Complutense de Madrid y Licenciada en Magisterio de Enseñanza Primaria. Actualmente trabaja como Profesora del Departamento de Psicología Diferencial y Ocupacional de esta universidad. Su investigación se centra en la evaluación de la carga de trabajo. Dos de sus principales publicaciones son: «Desarrollo del cuestionario CarMen-Q para la evaluación de la carga de trabajo mental» (Psicothema, 2017); y "Contexto académico y carga de trabajo mental percibida de los estudiantes de psicología», (Spanish Journal of Psychology, 2014).

iD https://orcid.org/0000-0003-2422-4458

Eva María Díaz-Ramiro es Doctora en Psicología y Profesora en el Departamento de Psicología Social, del Trabajo y Diferencial de la Universidad Complutense de Madrid. Dos de sus principales 
publicaciones son: «Desarrollo del cuestionario CarMen-Q para la evaluación de la carga de trabajo mental» (Psicothema, 2017) y «Contexto académico y carga de trabajo mental percibida de los estudiantes de psicología» (Spanish Journal of Psychology, 2014).

(iD https://orcid.org/0000-0002-7551-7915 


\section{revista española de pedagogía año 77, $n^{\circ}$ 272, enero-abril 2019}

Spanish Journal of Pedagogy

year 77, n. 272, January-April 2019

Sumario*

Table of Contents **

\section{Estudios \\ Studies}

Francisco López Rupérez, Isabel Garcia García,

\section{Eva Expósito Casas}

Rendimiento en ciencias, concepciones epistémicas y vocaciones STEM en las comunidades autónomas españolas. Evidencias desde PISA 2015, políticas y prácticas de mejora

Performance in Sciences, epistemic conceptions and STEM vocations in the Spanish Autonomous Communities. Evidence from PISA 2015, improvement policies and practices

\section{Zaida Espinosa Zárate}

El cultivo de la creatividad para el diálogo intercultural

Cultivating creativity for intercultural dialogue

Ignasi de Bofarull

Carácter y hábitos para el aprendizaje:

definición y proyecto de medición

Character and learning habits:

definition and measurement proposal

\section{9}

David Luque

Desarrollos interpretativos de la filosofía

de la educación en la tradición anglófona:

un intento de sistematización

Interpretive developments of the philosophy

of education in the anglophone tradition:

an attempt to systematise them

\section{Notas \\ Notes}

\section{María Moralo, Manuel Montanero}

Aprendizaje con y sin error en estudiantes con TEA

9 Learning with and without errors in students with ASD

85

\section{Antonio Portela Pruaño,}

José Miguel Nieto Cano, Ana Torres Soto

La reincorporación formativa de jóvenes

que abandonan tempranamente la educación:

47 relevancia de su trayectoria previa

Re-engagement in education and training

of young people who leave education early:

the importance of earlier prior trajectories

\footnotetext{
* Todos los artículos están también publicados en inglés en la página web de la revista: https://revistadepedagogia.org.

** All the articles are also published in English on the web page of the journal: https://revistadepedagogia.org.
} 
Oihane Fernández-Lasarte, Eider Goñi, Igor Camino, Estibaliz Ramos-Díaz

Apoyo social percibido e implicación escolar del alumnado de educación secundaria

Perceived social support and school engagement in secondary students

Jesús-Miguel Muñoz-Cantero, Luisa Losada-Puente

Validación del constructo de autodeterminación a través de la escala ARC.INICO para adolescentes Validation of the construct of self-determination through the ARC.INICO scale for teenagers

Ma Inmaculada López-Núnez, Susana Rubio-Valdehita, Eva Má Diaz-Ramiro

Cambios producidos por la adaptación al EEES en la carga de trabajo de estudiantes de Psicología Changes in Psychology students' workload due to alignment with the EHEA

\section{Reseñas bibliográficas}

Naval, C. y Arbués, E. (Eds.). Hacer la Universidad en el espacio social (Emanuele Balduzzi). Mínguez, R. y Romero, E. (Coords.). La educación ciudadana en un mundo en transformación: miradas y propuestas (Marina Pedreño Plana). Bellamy, F.-X. Los desheredados: por qué es urgente transmitir la cultura (Esteban López-Escobar). Pallarés, M., Chiva, 0., López Martín, R. y Cabero, I. La escuela que llega. Tendencias y nuevos enfoques metodológicos (Juan Carlos San Pedro Velado).

\section{Informaciones}

El Profesor Giuseppe Mari. In memoriam (Emanuele Balduzzi); III Conferencia Internacional de EuroSoTL: "Explorando nuevos campos a través de un enfoque académico de la enseñanza y el aprendizaje";

123 Una visita a la hemeroteca (Ana González-Benito); Una visita a la red (David Reyero).

\section{Instrucciones para los autores}

Instructions for authors 201 


\section{Changes in Psychology students' workload due to alignment with the EHEA}

\section{Cambios producidos por la adaptación al EEES en la carga de trabajo de estudiantes de Psicologia}

Ma Inmaculada LÓPEZ-NúÑEZ, PhD. Lecturer. Universidad Complutense de Madrid (mariai04@pdi.ucm.es).

Susana RUBI0-VALDEHITA, PhD. Lecturer. Universidad Complutense de Madrid (srubiova@ucm.es).

Eva Ma DÍAZ-RAMIRO, PhD. Assistant Professor. Universidad Complutense de Madrid (ediazram@ucm.es).

\section{Abstract:}

Alignment with the European Higher Education Area (EHEA) has involved changes in teaching methodology and in the learning process, which have led to a change in student workload. The main aim of this study is to evaluate and compare the perception of the workload of undergraduate and graduate psychology students and to analyse the nature of the changes produced by this adaptation. The sample comprised 845 students $(497$ readings for the licentiate's degree and 348 for the bachelor's degree). To measure perceived workload, the NASA-TLX scale was used. This evaluates the following dimensions: mental demand, physical demand, temporal demand, performance, and frustration/dissatisfaction; these variables were evaluated for the follow- ing academic activities: attending theoretical classes, attending practical classes, conducting group work outside the classroom, searching for material and bibliography, studying and personal work, attending tutorials, and other activities (seminars, conferences, etc.). The results show a significant increase in the perceived load of attendance in practical classes, in the performance of group work outside the classroom and in total perceived workload for bachelor's degree students. It is concluded that adjusting to the EHEA has involved an increase in perceived workload for students. This points to a need to work in the classroom on developing interpersonal skills and planning and organization of teamwork, as well as greater coordination between teachers in the evaluation systems.

Revision accepted: 2018-04-20.

This is the English version of an article originally printed in Spanish in issue 272 of the revista española de pedagogía. For this reason, the abbreviation EV has been added to the page numbers. Please, cite this article as follows: López-Núñez, M. I., Rubio-Valdehita, S., \& Díaz-Ramiro, E. (2019). Cambios producidos por la adaptación al EEES en la carga de trabajo de estudiantes de Psicología | Changes in Psychology students' workload due to alignment with the EHEA. Revista Española de Pedagogía, 77 (272), 163-176. doi: https://doi.org/10.22550/REP77-1-2019-02 
Keywords: European Higher Education Area, workload, NASA-TLX, students, psychology, higher education.

\section{Resumen:}

La adaptación al Espacio Europeo de Educación Superior (EEES) ha supuesto una importante modificación en la metodología docente y en el proceso de aprendizaje, lo que ha generado un cambio respecto a la carga de trabajo del alumnado. El principal objetivo de este estudio es evaluar y comparar la percepción de la carga de trabajo de estudiantes de Psicología de la licenciatura y del grado y analizar la naturaleza de los cambios producidos por esta adaptación. La muestra utilizada estuvo formada por 845 estudiantes (497 de licenciatura y 348 de grado). Para la medida de la carga percibida se empleó la escala NASA-TLX, que evalúa las siguientes dimensiones: demanda mental, demanda física, demanda temporal, rendimiento y frustración/insatisfacción; estas variables fueron evaluadas para las siguien- tes actividades académicas: asistencia a clases teóricas, asistencia a clases prácticas, realización de trabajos en grupo fuera del aula, búsqueda de material y bibliografía, estudio y trabajo personal, asistencia a tutorías y otras actividades (seminarios, jornadas, etc.). Los resultados muestran un aumento significativo en la carga percibida de la asistencia a clases prácticas, en la realización de trabajos en grupo fuera del aula y en la carga total percibida para los estudiantes de grado. Se concluye que la adaptación al EEES ha supuesto un incremento en la percepción de carga de trabajo de los estudiantes, lo que apunta a la necesidad de trabajar en las aulas el desarrollo de competencias interpersonales y de planificación y organización del trabajo en equipo, así como una mayor coordinación docente entre los sistemas de evaluación.

Descriptores: Espacio Europeo de Educación Superior, carga de trabajo, NASA-TLX, estudiantes, psicología, Educación Superior.

\section{Introduction}

The objectives of the European Higher Education Area (EHEA), which is the result of the Bologna Declaration (1999), include obtaining high employability rates, improving the competitivity of the higher education system in Europe, and promoting mobility with the ultimate aim of promoting a European dimension in the higher education system (Ariza, Quevedo-Blasco, Bermúdez,
$\&$ Buela-Casal, 2013). To achieve these objectives, a transformation in the structure of teaching was proposed through the European Credit Transfer and Accumulation System (ECTS), a system that distributes the student's workload over estimated hours, some face-to-face (classes, evaluations, etc.), some outside class with tutoring (guided work and tutorials) and some independent outside class (individual study and activities). 
The adjustment to the EHEA has involved important changes at the institutional level and in syllabuses (Ariza, Bermúdez, Quevedo-Blasco, \& Buela-Casal, 2012; Ortega \& Zych, 2013), and in the teaching methodologies used, with the incorporation of more active methods (such as attending seminars, solving problems as a team, doing projects and presentations, etc.) that give students more autonomy in a learning style with less face-to-face work and more personal work. These modifications also involve a change to a more constructivist teaching and learning process (Tejada \& López, 2012), focussed on the acquisition of competences and on continuous work by the student (De Miguel, 2006; Rodrigo \& Almirón, 2013), which involves a greater load of work outside class (Díez, García, \& IPDDA, 2010; Ortiz et al., 2012).

One of the fundamental issues in the new syllabuses is the students' workload (Roca-Cuberes, 2013). Workload can be defined as the hours students spend working on academic tasks at the educational centre (attending classes, tutorials, seminars) and away from it (groupwork, study, bibliographic consultations, etc.). In other words, it relates to the time required to complete an academic year and acquire the competences established as the learning outcomes.

The literature we have consulted agrees that adapting the workload to the credits established in the syllabuses is one of the main problems facing teaching staff (Reyes, Valdés, \& Castaño, 2006) and that the ECTS system involves an increase in student workload (Castaño, Ruiz, Gómez-Alday, \& De Manuel, 2006; Roca-Cuberes, 2013; Rodríguez-Izquierdo, 2014).

Although this educational reform was officially introduced in 2010, there are no empirical studies that analyse the effect of this adjustment from the students' perspective, especially in the case of teaching psychology (Ramiro-Sánchez, Bermúdez, \& Buela-Casal, 2016). Consequently, there is a clear lack of studies that explore the impact of these changes on the perceived workload of students who are reading for a bachelor's in psychology compared with those studying under previous system (licentiate degree). For example, Ortiz et al. (2012), from a perspective based on studying the time dedicated to each task, found differences in the workloads of licentiate and bachelor's degree students, and they note that the change in teaching-learning methodologies should be considered from the perspective of the students.

Students' perceived workload can have a very important influence on the level of academic performance they obtain and on their psychological wellbeing, as it is one of the main causal factors of academic stress (Cabanach, Souto-Gestal, \& Franco, 2016). Excess workload makes it hard to acquire knowledge and promotes superficial learning (Lam, McNaught, Lee, \& Chan, 2012; Roca-Cuberes, 2013), failure, and dropout (Cope \& Staehr, 2005).

Student workload was evaluated from different perspectives. It has traditionally been linked to how many hours students dedicate to the modules that make 
up their degree (Ruiz-Gallardo, Castaño, Gómez-Alday, \& Valdés, 2011), although in a small number of cases students' perception of their workload has also been analysed (Kyndt, Dochy, Struyven, \& Cascallar, 2011). This second focus assumes that students' perceived workload is shaped by various factors and that this perception is the largest stress creating factor (Ramsden, 1992). On these lines, Kember and Leung (2006) found that many hours of study are not perceived as a large overload when the course design is appropriate, and they underline the limited relationship between time spent on a task and perceived workload (Hertzum \& Holmegaard, 2013; Nosair \& Handy, 2017).

One of the most frequently used instruments for evaluating perceived workload is the NASA-Task Local Index (TLX) (Hart \& Staveland, 1988). González, Moreno, and Garrosa (2005), Hart (2006), and Young, Zavelina, and Hooper (2008) identify the useful features of NASA-TLX as a valid and reliable instrument for analysing perceived workload in different sectors: industrial, psychological, health, aeronautical, and transport. However, it has been used less in the field of education. López-Núnez (2010) analyses the generalisability and validity of each scale with a broad sample of university students and concludes that it is a useful instrument for evaluating workload in an educational setting. Kyndt et al. (2011) use NASA-TLX to evaluate the workload with the objective of analysing its mediating role in student motivation and their learning strategies. Similarly, Kurata,
Bano, and Matias (2015) use NASA-TLX on engineering students, finding significant relationship between workload and academic performance.

Almost two decades after the Bologna Declaration (1999) and in the midst of the changes to accreditation of qualifications adapted to the EHEA, this seems to be an appropriate time to evaluate the effects of this adaption. The change seems to have been positive in regards to the inclusion of new technology (ITC) and methodologies that permit greater interaction with students. However, other aspects such as increased workload, both for teaching staff and students, do not seem to be as positive (Ariza et al., 2013; Quevedo-Blasco, Ariza, \& Buela-Casal, 2015).

Therefore, the aim of this study is to analyse and compare the perceived workload of psychology students on the licentiate degree (old system) and of students on the new system adapted to the EHEA (bachelor's) to investigate the nature of the changes in this variable.

\section{Method \\ 2.1. Participants}

The sample comprised 845 third-year Psychology students from the Universidad Complutense de Madrid, 497 of whom were licentiate students and 348 bachelor's students. Regarding distribution by sex, there were 415 women on the licentiate degree $\left(83.5^{\circ} \%\right)$ and 82 men (16.5\%), and 282 women $(81 \%)$ and 66 men $(19 \%)$ on the bachelor's degree. The mean age of students on the licentiate degree was 21.63 
$(S D=3.33)$. On the bachelor's degree it was $21.85(S D=3.53)$. The data were collected in a single session during one of the third-year classes, for both the licentiate degree and the bachelor's degree, using convenience sampling.

\subsection{Instrument}

The NASA-TLX scale was used to assess workload (Hart \& Staveland, 1998). This instrument distinguishes six workload dimensions, each of which is evaluated by the subjects on a scale of 0 to 100 . These dimensions are: effort (the general level of mental and physical effort required to obtain a suitable level of performance), mental demand (the amount of mental and perceptive activity the task requires), physical demand (the amount of physical activity the task requires), temporal demand (the perceived level of temporal pressure), performance (the level of satisfaction with one's own level of performance in the task) and frustration/dissatisfaction (the level of insecurity, irritation, or dissatisfaction felt while performing the task). The students had to evaluate the degree to which each of their academic activities demanded each workload dimension from them. Seven academic activities were identified: attending theoretical classes, attending practical classes, conducting group work outside the classroom, searching for material and bibliography, studying and personal work, attending tutorials, and other activities (seminars, conferences, etc.).

These activities were chosen taking into account the changes imposed by the EHEA and the importance it gives to au- tonomous work by the student and the competence acquisition, including specific competences ones relating to the qualification and transversal ones, for students' integration into the social and workplace environment.

Using the evaluations given by each student, an overall workload index was calculated for each activity by calculating the arithmetic mean for the scores from each dimension (Hart, 2006).

Additionally, each participant answered a brief demographic questionnaire to collect information on sex and age.

\subsection{Design and Procedure}

This is a non-experimental, ex post facto study.

The NASA-TLX scale, in its implementation phase initially had two sections: a weighting section and a scoring section. The weighting section was intended to reveal the subjects' initial perceived workload and the relative importance of each of the six dimensions in this perception. However, many studies have underlined that it is not necessary to do the weighting stage (López, Rubio, Martín, \& Luceño, 2010), showing high correlation values between weighed and unweighted scores (between .93 and .97). For this reason, we only used the scoring section. All of the students took part anonymously and completely voluntarily, having first signed an informed consent form to agree to participate in the study and agreeing to the use of the data for research-related ends exclusively. 


\subsection{Statistical analysis}

All of the analysis was done using the Statistical Package for the Social Science (SPSS) version 22.00 software. Firstly, the descriptive statistics for all of the variables considered were calculated. Next, the equivalence of the two groups by average age and distribution by sex was tested using the $\chi^{2}$ statistic (for sex) and Student's $t$ test (for age). After this, a comparison of means analysis was performed on the workload scores obtained for both groups (licentiate degree vs. bachelor's) for each of the academic activities. To do this, Student's t value was calculated along with its level of significance $p$ (set at .05) and partial $\eta^{2}$ to test the effect size.

\section{Results}

Firstly, the means and standard deviations (SD) were calculated for the workload scores (for each workload dimension and for the overall score) in both groups (licentiate and bachelor's degrees) (Table 1). The total workload scores were obtained by averaging the workload scores for all of the dimensions and activities.

The results showed that the groups were equivalent in distribution by sex $\left(\chi^{2}=1.70, p=.430\right)$ and by mean age $[t(913)=-0.66, p=.508]$, as neither figure was significant.

Once compliance with the assumption of homogeneity of variance had been tested using Levene's test ( $P>0.5$ in all cases), comparisons of means for the overall scores for each activity were done for each type of degree. The results of this analysis are shown in Table 1. Analysing this table shows that bachelor's students perceive a higher workload than the ones on the licentiate degree in all of the activities considered other than «studying and personal work». The differences in the «conducting group work outside the classroom» and «attending practical classes» activities were statistically significant, as were the scores for overall workload. In the three activities, the group of students on the bachelor's degree displayed considerably higher workload scores. The $\eta^{2}$ values show that the effect size is higher for the "conducting group work outside the classroom» activity, indicating that the difference is most evident with this activity.

With the aim of analysing in greater detail which workload dimension or dimensions cause these differences, comparisons of means were carried out between bachelor's and licentiate degrees for each of the 6 dimensions of the NASA-TLX for the activities in which significant results were found in the previous analysis. The results of this analysis are shown in Table 2 .

For the «attending practical classes» activity, the results only show large and statistically significant differences in the mental demand dimension, indicating that bachelor's degree students regard this academic activity as rather more demanding from a cognitive perspective than their licentiate counterparts. For «conducting group work outside the classroom», the results show greater differences than for the previous activity. In this case, the differences between both groups were statis- 
tically significant in all of the dimensions have to make a greater effort to achieve except in performance. These results sug- very similar levels of performance. This in gest that doing group work outside class turn creates more marked feelings of frushours is a much more demanding activity tration. The results for the total workload at all levels for bachelor's students, who score are similar.

TABLE 1. Mean (M) and standard deviation (SD) for the overall workload for each activity and results of the comparison of means across qualifications.

\begin{tabular}{|c|c|c|c|c|c|}
\hline & Licentiate & Bachelor's & & & \\
\hline & $\mathrm{M}(\mathrm{DT})$ & $\mathrm{M}(\mathrm{DT})$ & $t(843)$ & $p$ & $\eta^{2}$ \\
\hline Attending theoretical classes & $\begin{array}{c}52.86 \\
(12.29)\end{array}$ & $\begin{array}{c}53.35 \\
(13.09)\end{array}$ & -0.55 & .581 & .000 \\
\hline Attending practical classes & $\begin{array}{c}47.80 \\
(13.82)\end{array}$ & $\begin{array}{c}49.80 \\
(13.00)\end{array}$ & -2.11 & .035 & .005 \\
\hline $\begin{array}{l}\text { Conducting group work outside } \\
\text { the classroom }\end{array}$ & $\begin{array}{c}49.63 \\
(14.81)\end{array}$ & $\begin{array}{c}55.01 \\
(13.44)\end{array}$ & -5.38 & .000 & .033 \\
\hline $\begin{array}{l}\text { Searching for material } \\
\text { and bibliography }\end{array}$ & $\begin{array}{c}40.28 \\
(15.23)\end{array}$ & $\begin{array}{c}40.62 \\
(14.51)\end{array}$ & -0.32 & .744 & .000 \\
\hline Studying and personal work & $\begin{array}{c}57.05 \\
(12.51)\end{array}$ & $\begin{array}{c}56.79 \\
(11.50)\end{array}$ & 0.31 & .756 & .000 \\
\hline Attending tutorials & $\begin{array}{c}25.68 \\
(11.82)\end{array}$ & $\begin{array}{c}26.97 \\
(12.87)\end{array}$ & -1.49 & .135 & .003 \\
\hline $\begin{array}{l}\text { Other activities } \\
\text { (seminars. conferences. etc.) }\end{array}$ & $\begin{array}{c}28.72 \\
(14.46)\end{array}$ & $\begin{array}{c}29.42 \\
(14.29)\end{array}$ & -0.68 & .497 & .001 \\
\hline Total workload & $\begin{array}{l}43.37 \\
(9.20)\end{array}$ & $\begin{array}{l}44.58 \\
(8.96)\end{array}$ & -1.89 & .048 & .005 \\
\hline
\end{tabular}

Source: Own elaboration. 
TABLE 2. Mean (M), standard deviation (SD) and results of the comparison of means between both qualifications in workload scores by NASA-TLX dimension.

Attending

practical classes

Conducting group

work outside the

classroom

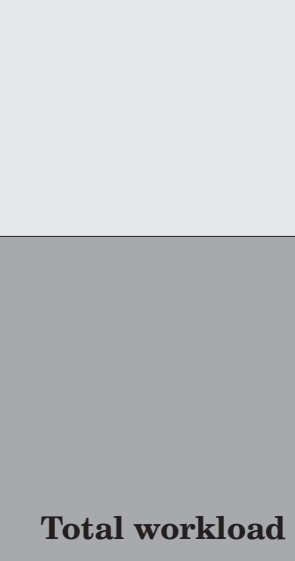

\section{Total workload}




\section{Discussion}

Given the lack of research that has examined student perceptions of the academic demands resulting from adapting degrees to the EHEA, this study aimed to evaluate workload levels in two samples of psychology students (from bachelor's and licentiate degree programmes) and analyse whether the changes introduced by adapting to the EHEA have caused any significant change in student's perceived workload.

Using the NASA-TLX scale not only made it possible to discover the workload perceived by the students, but also to go into more depth in the understanding which activities generate the highest workload levels.

The results of this study indicate that there are differences in the total workload perceived by each group, with higher scores obtained from the bachelor's group (adapted to the EHEA) than the licentiate group (old system). Taking specific tasks into account, statistically significant differences were found for the «attending practical classes» and "conducting group work outside the classroom» activities.

Analysing each of them in greater detail, we find that for the «attending practical classes» task there are statistically significant differences in the mental demand dimension and so this activity requires a greater cognitive effort from the bachelor's group than from the licentiate group. The «conducting group work outside the classroom» activity is perceived as being far more demanding than the previous one, with significant differences found in all dimensions apart from performance. This means that bachelor's students have to make greater efforts to achieve similar results, which as well as making greater demands also causes feelings of frustration.

These results allow us to draw two important conclusions from the adjustment to the EHEA, conclusions that require reflection and open up perspectives for future research. The first result is proof that the students perceive a greater workload in the new psychology degree since the implementation of the bachelor's programme; the second one shows that the activity that creates the greatest load in all dimensions is "conducting group work outside the classroom».

With regards to the first question, the results found coincide with those obtained by other authors regarding an increase in perceived workload in bachelor's students compared with those on the licentiate degree (Roca-Cuberes, 2013; Rodríguez-Izquierdo, 2014). To explain this result, various pieces of research state that the workload in bachelor's qualifications is no higher than the corresponding workload according to number of credits and that the increase in workload perceived by the students could be because the new methodologies require a continuous temporal distribution throughout the term (Julián et al., 2010). Other authors state that the lack of a relationship between effort and performance is not 
explained by a greater workload, but instead by the lack of appropriate study techniques, limited use made of learning resources such as personal tutoring, and the need for greater effort from students (Ortíz et al., 2012). This increase in perceived workload could be because the EHEA establishes an autonomous teaching and learning system that requires organisation and planning strategies that are unfamiliar to students and for which they are unprepared.

Future research should evaluate the current perception of workload and perform longitudinal studies to discover whether the new bachelor's degrees are resulting in the acquisition of more proactive learning and a reduction in perceived workload over academic years. Their results would make it possible to discover what the process of adaptation to the EHEA has been like in order to propose interventions in study strategies and skills, applying knowledge from pedagogy with the aim of facilitating and improving the learning process.

As for the second consideration, namely that "conducting group work outside the classroom» is the activity that produces the greatest perceived load, this would indicate the difficulty students have in adapting to a new system of learning that involves developing interpersonal and planning and organisational competences. In addition, this is identified as a stressful activity that requires time and where the greater effort does not correspond with the expected reward.
The EHEA identifies team work as one of the principal transversal competences. Several studies (Alonso-Martín, 2010; Arce, Fariña, Novo, \& Seijo, 2012; París, Torrelles, \& Mas, 2016) have evaluated the acquisition and development of this competence in university students. The results of this research show that students do not regard this competence as a resource that helps them with their learning, but they do perceive it as a stressful factor that, on the whole, students are not acquiring. As Fidalgo-Blanco, Leris, Sein-Echaluce, and Garcia-Peñalvo (2013) observe, in the academic setting, teamwork is usually applied using what they call a «black box» model. In other words, it is not the teamwork competence of individuals that is assessed, but instead just the quality of the work once it is completed. Consequently, students are left to develop group-work skills through mere exposure, without giving them any resources or guidance, erroneously assuming that the students start university courses having already acquired this skill in non-university education.

The results found in this study make apparent the need to develop training programmes for students and for teachers to develop and evaluate this transversal competence, as it is precisely one of the most important of those identified in the professional profile of psychology graduates (ANECA, 2005).

With regards to the limitations of the study, it should be noted that it is an incidental sample from a single university, and a larger sample from several 
Spanish universities would have allowed for more generalisable results to be obtained.

Finally, among the main practical implications of the results obtained, it is worth noting that this is a pioneering study in the evaluation of workload levels in the move towards the bachelor's degree in psychology in the EHEA. In agreement with the literature consulted, it is confirmed that this adaptation entailed an increase in perceived student workload levels and that the group work activity is perceived as especially demanding and frustrating. These results make it possible to open up new lines of research concerning the differences detected and propose future studies, considering perceived workload as an indicator of quality and of evaluation of adjustment to the EHEA.

\section{References}

Agencia Nacional de Evaluación de la Calidad y Acreditación, ANECA (2005). Libro blanco del título de grado en Psicología. Retrieved from https://goo.gl/Lucfeq (Consulted on 29/3/2017).

Alonso-Martín, P. (2010). La valoración de la importancia de las competencias transversales: comparación de su percepción al inicio y final de curso en alumnos de psicología. Revista de investigación educativa, 28 (1), 119-140.

Arce, R., Fariña, F., Novo, M., \& Seijo, D. (2012). Efecto del sistema de enseñanza en el rendimiento académico, burnout experimentado y estrés académico. Aula abierta, 40 (2), 3-10.

Ariza, T., Bermúdez, M. P., Quevedo-Blasco, R., \& Buela-Casal, G. (2012). Evolución de la legislación de doctorado en los países del EEES. Revista Iberoamericana de Psicología y Salud, 3 (2), 89-108.
Ariza, T., Quevedo-Blasco, R., Bermúdez, M. P., \& Buela-Casal, G. (2013). Analysis of postgraduate programs in the EHEA and the USA. Revista de Psicodidáctica, 18 (1), 197-219. doi: https:// doi.org/10.1387/RevPsicodidact.5511

Cabanach, R. G., Souto-Gestal, A., \& Franco, V. (2016). Escala de Estresores Académicos para la evaluación de los estresores académicos en estudiantes universitarios. Revista Iberoamericana de Psicología y Salud, 7 (2), 41-50.

Castaño, S., Ruiz, J. R., Gómez-Alday, J. J., \& De Manuel, T. (2006). Adaptación metodológica al EEES: resultados de una experiencia. Revista de la Red-U, 5 (1), 35-48.

Cope, C., \& Staehr, L. (2005). Improving students' learning approaches through intervention in an information systems learning environment. Studies in Higher Education, 30 (2), 181-197. doi: https:/doi. org/10.1080/03075070500043275

De Miguel, M. (Ed.). (2006). Metodología de enseñanza y aprendizaje para el desarrollo de competencias. Madrid: Alianza editorial.

Bologna Declaration (1999). El espacio Europeo de la enseñanza superior. Declaración conjunta de los ministros europeos de educación. Retrieved from https://goo.gl/aoXldD (Consulted on 20/3/2017).

Díez, M. C., García, J. N., \& IPDDA. (2010). Percepción de metodologías docentes y desarrollo de competencias al EEES. Boletín de Psicología, 99, 45-69.

Fidalgo-Blanco, Á., Lerís, D., Sein-Echaluce, M. L., \& García-Peñalvo, F. J. (2013). Indicadores para el seguimiento y evaluación de la competencia de trabajo en equipo a través del método CTMTC. Trabajo presentado en el II Congreso Internacional sobre Aprendizaje, Innovación y Competitividad (CINAIC 2013). Retrieved from https://goo.gl/1aiZBq (Consulted on 19/9/2017).

González, J. L., Moreno, B., \& Garrosa, E. (2005). Carga mental y fatiga laboral. Madrid: Pirámide. 
Hart, S. G. (2006). NASA-Task Load Index (NASA-TLX); 20 years later. Proceedings of the Human Factors and Ergonomics Society Annual Meeting, 50 (9), 904-908. doi: https://doi. org/10.1177/154193120605000909

Hart, S. G., \& Staveland, L. E. (1988). Development of NASA-TLX (Task LoadIndex): Results of empirical and theoretical research. In P. A. Hancock \& N. Meshkati (Eds.), Human mental workload (pp. 139-183). Amsterdam: North-Holland.

Hertzum, M., \& Holmegaard, K. D. (2013). Perceived time as a measure of mental workload: Effects of time constraints and task success. International Journal of Human-Computer Interaction, 29 (1), 26-39. doi: https://doi.org/10.1 080/10447318.2012.676538

Julián, J., Zaragoza, J., Castejón, F. J., \& López, V. M. (2010). Carga de trabajo en diferentes asignaturas que experimentan el sistema ECTS. Revista Internacional de Medicina y Ciencias de la Actividad Fisica y del Deporte, 10 (38), 218-233.

Kember, D., \& Leung, D. Y. (2006). Characterising a teaching and learning environment conducive to making demands on students while not making their workload excessive. Studies in Higher Education, 31 (2), 185-198.doi: https:// doi.org/10.1080/03075070600572074

Kyndt E., Dochy F., Struyven K., \& Cascallar E. (2011). The direct and indirect effect of motivation for learning on students' approaches to learning through the perceptions of workload and task complexity. Higher Education Research \& Development, 30 (2), 135-150. doi: https://doi.org/10.1080/07294360.2010.501329

Kurata, Y. B., Bano, R. M. L. P., \& Matias, A. C. (2015). Effects of workload on academic performance among working students in an undergraduate engineering program. Procedia Manufacturing, 3, 3360-3367. doi: https://doi. org/10.1016/j.promfg.2015.07.497

Lam, P., McNaught, C., Lee, J., \& Chan, M. (2012). The impact of student workload on learning experiences. Hong Kong: Centre for Learning Enhancement and Research, the Chinese Uni- versity of Hong Kong. Retrieved from https:// goo.gl/LCg2G8 (Consulted on 2/10/2017)

López, I., Rubio, S., Martín, J., \& Luceño, L. (2010). Fase de ponderación del NASA-TLX: iun paso innecesario en la aplicación del instrumento? Edupsykhé: Revista de Psicología y Educación, 9 (2), 159-176.

López-Núñez, M. I. (2010). Generalización al ámbito laboral de dos instrumentos de medida subjetiva de la carga mental (Doctoral thesis). Universidad Complutense de Madrid. Retrieved from https://goo.gl/MPIBKT (Consulted on 26/4/2017).

Nosair, E., \& Hamdy, H. (2017). Total Student Workload: Implications of the European Credit Transfer and Accumulation System for an Integrated, Problem-Based Medical Curriculum. Health Professions Education, 3 (2), 99-107. doi: https://doi.org/10.1016/j.hpe.2017.01.002

Ortega, R., \& Zych, I. (2013). Profesionales de la Psicología y formación universitaria: buscando referencias para el título de grado en Psicología. International Journal of Psychology and Psychological Therapy, 13 (1), 83-96.

Ortíz, V. M., Jenaro, C., Meilán, J. G., Zubiauz, B., Mayor, M. A., \& Arana, J. M. (2012). Carga de Trabajo en el EEES: La necesidad de coordinación docente entre asignaturas. In J. D. Álvarez, M. T. Tortosa, \& N. Pellín (Coords.), IX Jornadas de Redes de Investigación en Docencia Universitaria. Diseño de buenas prácticas docentes en el contexto actual, 1863-1877. Retrieved from https://goo.gl/THFgLK

París, G., Torrelles, C., \& Mas, O. (2016). La evaluación de la competencia «trabajo en equipo» de los estudiantes universitarios. Revista d'Innovació Docent Universitària, 8, 86-97. doi: https://doi.org/10.1344/RIDU2016.8.10

Quevedo-Blasco, R., Ariza, T., \& Buela-Casal, G. (2015). Evaluación de la satisfacción del profesorado de ciencias con la adaptación al Espacio Europeo de Educación Superior. Educación XXI, 18 (1), 45-70. doi: https://doi.org/10.5944/ educXX1.18.1.12311 
Ramiro-Sánchez, T., Bermúdez, M. P., \& Buela-Casal, G. (2016). Titulación de Psicología en el EEES: comparación entre licenciados y graduados españoles. Revista de Psicodidáctica, 21 (1), 175-189. doi: https://doi.org/10.1387/ RevPsicodidact.13923

Ramsden P. (1992). Learning to teach in higher education. London, UK: Routledge.

Reyes, J. R., Valdés, A., \& Castaño, S. (2006). Prácticum y carga de trabajo. Revista deInvestigación Educativa, 24 (2), 557-574.

Rodríguez-Izquierdo, R. M. (2014). Modelo formativo en el Espacio Europeo de Educación Superior: valoraciones de los estudiantes. Aula Abierta, 42 (2), 106-113. doi: https://doi. org/10.1016/j.aula.2014.03.002

Roca-Cuberes, C. (2013). La percepción de los estudiantes sobre su experiencia de aprendizaje a partir de su dedicación temporal y adquisición de competencias: un estudio sobre la implementación del Grado de Traducción e Interpretación de la Universidad Pompeu Fabra. Revista Complutense de Educación, 24 (2), 359-379.

Rodrigo, M., \& Almiron, N. (2013). Autopercepción de la adquisición de competencias de los estudiantes de periodismo: el caso de la Universitat Pompeu Fabra. Aula Abierta, 41 (1), 99-110.

Ruiz-Gallardo J. R., Castaño, S., Gómez-Alday J. J., \& Valdés, A. (2011). Assessing student workload in problem based learning: relationships among teaching method, student workload and achievement. A case study in Natural Sciences. Teaching and Teacher Education, 27 (3), 619-627. doi: http://doi.org/10.1016/j. tate.2010.11.001

Tejada, Á., \& López, M. (2012). Nuevas metodologías docentes en los títulos de grado: la literatura como recurso pedagógico colaborativo. Aula Abierta, 40 (3), 107-114.

Young, G., Zavelina, L., \& Hooper, V. (2008). Assessment of workload using NASA task load index in perianesthesia nursing. Journal of Perianesthesia Nursing, 23 (2), 102-110. doi: https://doi. org/10.1016/j.jopan.2008.01.008

\section{Authors' biographies}

María Inmaculada López-Núñez is a PhDin Psychology from the Universidad Complutense de Madrid and a Lecturer in the Social, Occupational and Differential Psychology Department at this university. She teaches in the Bachelor's Degree in Psychology, and on the Master's in Teacher Training, in the Occupational Training and Orientation speciality in the Faculty of Education. Her main lines of research: occupational psychology, professional competence development and acquisition, innovation, and entrepreneurship.

(D) https://orcid.org/0000-0001-9179-7219

Susana Rubio-Valdehita has a $\mathrm{PhD}$ in Psychology and is graduated in Primary School Teaching. She works as a Lecturer and Head of the Social, Occupational and Differential Psychology Department at the Universidad Complutense de Madrid. Her research focuses on evaluating workload. Selected publications: «Development of the CarMen-Q Questionnaire for mental workload assessment» (Psicothema, 2017), and «Academic context and perceived mental workload of psychology students» (Spanish Journal of Psychology, 2014).

(D) https://orcid.org/0000-0003-2422-4458

Eva María Díaz-Ramiro has a PhD in Psychology and is a Professor in the Social, Occupational and Differential Psychology Department at the Universidad Complutense de Madrid. Selected publications: «Development of the CarMen-Q 
Questionnaire for mental workload assessment» (Psicothema, 2017) and «Academic context and perceived mental workload of psychology students» (Spanish Journal of Psychology, 2014).

iD https://orcid.org/0000-0002-7551-7915 


\section{revista española de pedagogía año 77, n 272, enero-abril 2019 \\ Spanish Journal of Pedagogy year 77, n. 272, January-April 2019 \\ Table of Contents \\ Sumario}

\section{Studies}

\section{Estudios}

Francisco López Rupérez, Isabel García García,

\section{Eva Expósito Casas}

Performance in science, epistemic conceptions, and STEM vocations in Spain's autonomous communities: evidence from PISA 2015, improvement policies, and practices Rendimiento en Ciencias, concepciones epistémicas y vocaciones STEM en las comunidades autónomas españolas. Evidencias desde PISA 2015, políticas y prácticas de mejora $\mathbf{5}$

\section{Zaida Espinosa Zárate}

Cultivating creativity for intercultural dialogue

El cultivo de la creatividad para el diálogo intercultural

Ignasi de Bofarull

Character and learning habits:

definition and measurement proposal

Carácter y hábitos para el aprendizaje:

definición y proyecto de medición

David Luque

Interpretive developments of the philosophy of education in the anglophone tradition: an attempt to systematise them Desarrollos interpretativos de la filosofía de la educación en la tradición anglófona: un intento de sistematización

\section{Notes}

Notas

María Moralo, Manuel Montanero

Learning with and without errors in students with ASD Aprendizaje con y sin error en estudiantes con TEA

Antonio Portela Pruaño, José Miguel Nieto Cano, Ana Torres Soto

Re-engagement in education and training of young people who leave education early: the importance of earlier prior trajectories

La reincorporación formativa de jóvenes

que abandonan tempranamente la educación:

relevancia de su trayectoria previa

Oihane Fernández-Lasarte, Eider Goñi, Igor Camino,

Estibaliz Ramos-Díaz

Perceived social support and school engagement in secondary students

47 Apoyo social percibido e implicación escolar del alumnado de educación secundaria

Jesús-Miguel Muñoz-Cantero, Luisa Losada-Puente Validation of the construct of self-determination through the ARC.INICO scale for teenagers Validación del constructo de autodeterminación a través de la escala ARC.INICO para adolescentes 


\section{Månmaculada López-Núñez, Susana Rubio-Valdehita,}

Eva Ma Diaz-Ramiro

Changes in Psychology students' workload due to

alignment with the EHE

Cambios producidos por la adaptación al EEES

en la carga de trabajo de estudiantes de Psicología

\section{Book reviews}

Naval, C., \& Arbués, E. (Eds.). Hacer la Universidad

en el espacio social [Constructing the university in the

social space] (Emanuele Balduzzi). Mínguez, R., \&

Romero, E. (Coords.). La educación ciudadana en

un mundo en transformación: miradas y propuestas

[Citizenship education in a changing world:

perspectives and proposals] (Marina Pedreño Plana).

Bellamy, F.-X. Los desheredados: por qué es urgente

transmitir la cultura [The disinherited: why transmitting

culture is urgent] (Esteban López.Escobar). Pallarés,

M., Chiva, O., López Martín, R., \& Cabero, I. La

escuela que llega. Tendencias y nuevos enfoques

metodológicos [The school of the future: Trends and

new methodological focuses] (Juan Carlos San Pedro

Velado).

This is the English version of the research articles and book reviews published originally in the Spanish printed version of issue 272 of the revista española de pedagogía. The full Spanish version of this issue can also be found on the journal's website http://revistadepedagogia.org.

ISSN: 0034-9461 (Print), 2174-0909 (Online)

https://revistadepedagogia.org/

Depósito legal: M. 6.020 - 1958 